\title{
SESSION 5: Intestinal immunology
}

\section{Gut reaction: Immune pathways that control intestinal homeostasis}

Fiona Powrie

William Dunn School of Pathology, University of Oxford, South Parks Road, Oxford, UK

Please see the following reference for a recent review:

Maloy KJ \& Powrie F (2011). Intestinal homeostasis and its breakdown in inflammatory bowel disease. Nature; 474, 298-306 


\title{
Suppression of biofilms formation of enterotoxigenic Staphylococcus and Enterobacteriaceae by Lactobacillis bacteriocin-producing strain
}

\author{
Oxana V. Rybalchenko, ${ }^{\mathrm{a}, \mathrm{b}}$, Viktor M. Bondarenko ${ }^{\mathrm{a}}$, Olga G. Orlova ${ }^{\mathrm{a}, \mathrm{b}}$, Alexandra V. \\ Ryzhankova $^{a}$ \\ ${ }^{a}$ St. Petersburg State University, Russia; ${ }^{\mathrm{b}}$ Institute of Highly Pure Biopreparations, St. Petersburg, Russia
}

Aim. Investigation of bacterial biofilms suppression of enterotoxigenic Staphylococcus aureus and Enterobateriacae of different taxonomic groups by bacteriocin-producing Lactobacillus strain.

Materials and methods. The bacteriocin-producing strain Lactobacillus fermentum 97; target strains including Staphylococcus aureus, producing STA; LT-enterotoxigenic Citrobacter freundii, Enterobacter cloaceae, Klebsiella oxytoca, Proteus mirabilis, carrying the genes elt, identified by PCR analysis, and clinical strain of Candida albicans were used.

The effect of destruction of bacterial biofilms was determined using a deferred antagonism assay on solid medium. To examine the effects of biofilms disruption the ultrastructural changes of target cells compared with control.

Results. It was demonstrated that lactobacilli metabolites can inhibit the formation of staphylococci linked cellular structures that form the different space-oriented conglomerates of cells. After all they were transformed into the groups of separated and damaged bacterial cells. The interaction of lactobacilli metabolites to enterotoxigenic enterobacteria morphological features suppression of biofilms formation and disruption of the formed bacterial biofilms were observed.

Electron microscopy investigation revealed features of cell-cell interactions in the mixed culture of bacteriocin-producing strain of lactobacilli and sensitive to their antagonistic action cells of opportunistic (conditionally pathogenic) microorganisms of different taxonomic groups. The main characteristic of bacterial biofilms is membrane vesicles on the surface of cells and in the structures of surface biofilms. Potentially this vesicles may contain hydrolytic enzymes, moving to the surface of bacterial biofilms.

Conclusion. Our data clearly demonstrate that lactobacilli bacteriocin-producing substances suppress such processes as growth and development of number of opportunistic bacteria and microscopic fungi was shown. This effects of lactobacilli metabolites containing bacteriocins, approved in the inhibiting or disrupting of the bacterial biofilms formation of opportunistic enterotoxigenic strains of Enterobacteriaceae and Staphylococcus aureus. 


\title{
CD97 overexpression in enterocytes induces a megaintestine
}

\author{
Gabriela Aust ${ }^{1}$, Christiane Kerner ${ }^{1}$, Susann Gonsior ${ }^{1}$, Doreen Sittig ${ }^{1}$, Susanne M. Krug ${ }^{2}$, \\ Salah Amasheh ${ }^{2}$ \\ ${ }^{I}$ Department of Surgery, Research Laboratories, University of Leipzig, Germany \\ ${ }_{2}^{2}$ Institute of Clinical Physiology, Charité, Campus Benjamin Franklin, Berlin, Germany
}

Introductions and aims: CD97, an adhesion G protein-coupled receptor, is located in normal human enterocytes within adherens junctions. In transgenic ( $\mathrm{Tg}$ ) mice overexpressing the molecule in intestinal epithelial cells, CD97 strengthened these cell contacts (PloS one 2010;5:e8507). As we describe here, the $\mathrm{Tg}$ mice showed a selective enlargement of the small intestine. To clarify the underlying mechanism we analyzed this unexpected phenotype from various angles regarding histology, physiology and cell biology.

Methods and results: The increase of length and diameter of the small intestine corresponded to the CD97 cDNA copy number integrated. The colon was not affected. Living pups developed normally. In adult $\mathrm{Tg}$ mice microscopic intestinal architecture and number and position of proliferating and apoptotic intestinal cells were comparable to wild-type (WT) mice. Barrier properties as analyzed by measurement of epithelial resistance, dilution potentials and fluxes of paracellular markers yielded no differences compared to WT mice. Recently generated Tg mice, overexpressing CD97 truncated in the seven-transmembrane domains (TM7) to TM2, showed also lengthening of the small intestine indicating the impact of the $\alpha$-chain of CD97 for the effect.

Interestingly, the phenotype is acquired after birth during the postnatal period before weaning. Serum levels of growth factors, as growth hormone (GH), insulin-like growth factor (IGF)-1 and -2, and IGFbinding protein-3 (IGFBP-3), all known to cause intestinal lengthening in several models, were not different between postnatal and adult Tg and WT mice. Because lactoferrin, a milk constituent, was shown to induce small intestinal lengthening, Tg and WT mice were crossed and the resulting pups were nursed by a Tg mother. Only Tg pups showed intestinal lengthening suggesting that milk is not responsible for the effect. The murine lactoferrin receptor $(\mathrm{mLfR})$ is expressed at high levels in the small, not the large bowel. However, we did not found increased mLfR mRNA levels in the small intestine of $\mathrm{Tg}$ mice.

Conclusion: Transgenic villin-CD97 mice show a selective increase in length and diameter of the small intestine that is not caused by a selective upregulation of growth factors and their receptors. 